\section{Complex degree of mutual polarization of biological tissue coherent images for the diagnostics of their physiological state}

\author{
Oleg V. Angelsky \\ Alexander G. Ushenko* \\ Yevheniya G. Ushenko \\ Chernivtsi National University \\ Correlation Optics Department \\ 2 Kotsyubinsky Street \\ Chernivtsi, Ukraine 58012
}

\begin{abstract}
This is the first attempt devoted to the 1 st -4 th order statistic analysis of the complex degree of mutual polarization of biological tissues coherent images. The ensemble of diagnostic criteria (skewness and kurtosis of two-dimensional distributions of complex degree of mutual polarization) of pathological changes of muscular and connective (skin derma) tissues is defined. (๑) 2005 Society of Photo-Optical Instrumentation Engineers. [DOI: 10.1117/1.2149844]
\end{abstract}

Keywords: complex degree of mutual polarization; biological tissue; statistics.

Paper 05140LRR received Jun. 14, 2005; revised manuscript received Oct. 19, 2005; accepted for publication Oct. 28, 2005; published online Dec. 29, 2005.

\section{Introduction}

Real object fields scattered on biological tissue (BT) possess a change of both polarization and correlation characteristics. ${ }^{1}$ For analyzing these fields a new methodological approach is proposed $^{2-6}$ based on the analysis of complex degree of mutual polarization (CDMP) $V^{2}\left(r_{i}, r_{i+1}\right)$ of BT speckle image, determined by its magnitude $\left|V^{2}\left(r_{i}, r_{i+1}\right)\right|$ and opticalgeometric phase difference $\beta$ between the oscillations in the points $r_{i}$ and $r_{i+1}{ }^{6}$ :

$$
V^{2}\left(r_{i}, r_{i+1}\right)=\left|V^{2}\left(r_{i}, r_{i+1}\right)\right| \exp (-i \beta)
$$

where

$$
\begin{aligned}
\left|V^{2}\left(r_{i}, r_{i+1}\right)\right|= & \left(\left\{\left[I^{(0)}\left(r_{i+1}\right) I^{(0)}\left(r_{i}\right)\right]^{1 / 2}-\left[I^{(90)}\left(r_{i+1}\right) I^{(90)}\left(r_{i}\right)\right]^{1 / 2}\right\}^{2}\right. \\
& +4\left[I^{(0)}\left(r_{i+1}\right) I^{(90)}\left(r_{i+1}\right) I^{(0)}\left(r_{i}\right) I^{(90)}\left(r_{i}\right)\right]^{1 / 2} \\
& \left.\times \cos \left(\delta_{i+1}-\delta_{i}\right)\right) /\left(I\left(r_{i+1}\right) I\left(r_{i}\right)\right)
\end{aligned}
$$

Here $I^{(0)}\left(r_{i}\right) ; I^{(90)}\left(r_{i}\right)$ are arrays of the BT image intensities measured for passing axis orientation of the analyzer corresponding to the angles $\Theta=0 \mathrm{deg}$ and $\Theta=90 \mathrm{deg} ; \delta_{i+1}-\delta_{i}$ is the phase difference between the orthogonal components of oscillations $E^{(0)}\left(r_{i}\right), E^{(90)}\left(r_{i}\right)$ and $E^{(0)}\left(r_{i+1}\right), E^{(90)}\left(r_{r+1}\right)$ in the

*Tel: +382372244730; E-mail: ushenko-bio@itf.cv.ukrtel.net points with the corresponding coordinates $r_{i+1}$ and $r_{i}$ in the image plane.

From the physical point of view the CDMP 2-D distributions $V^{2}\left[I^{(0)}\left(r_{i+1}, r_{i}\right) ; I^{(90)}\left(r_{i+1}, r_{i}\right) ; \delta_{i+1}, \delta_{i}, \beta\right]$ characterize the degree of correlation between the states of the field polarization in its different points. Naturally this degree of correlation is determined by optical and structural characteristics of the object such as anisotropy, birefringence, etc. Pathological changes of BT are accompanied by the transformation of such a structure. ${ }^{7,8}$ Thus the analysis of physiologically normal and pathologically changed BT by means of 2-D distributions of CDMP proves to be important.

The parameter equal to that of visibility of interference pattern (which is formed by the beams with the polarization under consideration) was the base of metrological estimation of polarization interconnection in various points of the field. ${ }^{6}$ Decomposition into two mutually-orthogonal, linearlypolarized states with the corresponding oscillations phase shift $\delta_{1}, \delta_{2}$ was used for representing the arbitrary type of polarization. The measured visibility is a sum of additive partial visibilities of interference patterns formed by mutually parallel linear polarization components of two beams. ${ }^{9}$ The phase difference of oscillations in the orthogonally-polarized components forming two systems of interference beams, i.e., $\left(\delta_{i+1}-\delta_{i}\right)$ will show in relative shift of the interference pattern. This will also influence the resulting visibility of the whole interference pattern.

In this situation only the first of all CDMP phase components, $\left(\delta_{i+1}-\delta_{i} \equiv \delta_{i+1, i}\right.$ and $\left.\beta\right)$, has the diagnostic meaning. It is univocally interrelated with azimuths $\alpha\left(r_{i+1} ; r_{i}\right)$ and ellipticities $\gamma\left(r_{i+1} ; r_{i}\right)$ distributions of polarization images by the following relation ${ }^{1}$ :

$$
\begin{aligned}
\delta_{i+1, i}= & \operatorname{arctg}\left\{\operatorname{tg} 2 \gamma\left(r_{i+1}\right)\left[\sin 2 \alpha\left(r_{i+1}\right)\right]^{-1}\right\} \\
& -\operatorname{arctg}\left\{\operatorname{tg} 2 \gamma\left(r_{i}\right)\left[\sin 2 \alpha\left(r_{i}\right)\right]^{-1}\right\} .
\end{aligned}
$$

Sensitivity of the measuring polarization parameters is $1^{\prime}$. The phase $\beta$ of CDMP defines only the shift of the resulting interference pattern as a whole in the registration plane. It is not diagnostically important in this case.

The optical scheme for experimental measuring of CDMP of BT images is presented in Fig. 1. Illumination was realized by a collimated $\left(\varnothing=10^{4} \mu \mathrm{m}\right)$ He-Ne laser beam $(\lambda$ $=0.6328 \mu \mathrm{m}, W=5.0 \mathrm{~mW})$. The polarization illuminator consisted of two quarter-wave plates and a polarizer forming an illuminating beam with the range of azimuth $0 \mathrm{deg} \leqslant \alpha_{0}$ $\leqslant 180 \mathrm{deg}$ and with the range of polarization ellipticity $0 \mathrm{deg} \leqslant \gamma_{0} \leqslant 90 \mathrm{deg}$. BT polarization images were projected onto the plane of a light-sensitive area $(800 \times 600$ pixels $)$ of a CCD camera by means of a microscope objective. The CCD camera provided a measuring range of structural BT elements for the scales 2-2000 $\mu \mathrm{m}$. The polarization analysis of BT images was realized by using a polarizer. By rotating passage axis $\Theta$ of the analyzer within $\Theta=0 \mathrm{deg}-180 \mathrm{deg}$ one can define arrays of $\alpha\left(r_{i}\right), \gamma\left(r_{i}\right)$.

1083-3668/2005/10(6)/060502/3/\$22.00 @ 2005 SPIE 


\section{JBO LETTERS}

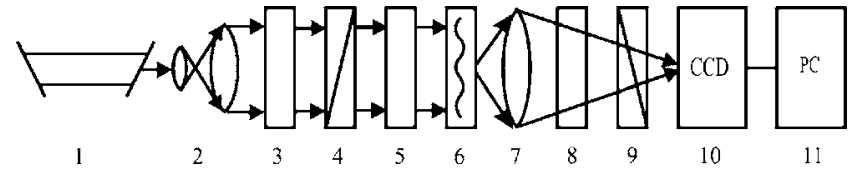

Fig. 1 Experimental setup. 1-He-Ne laser; 2-collimator; 3, 5, 8-quarter-wave plates; 4, 9-polarizer and analyzer, respectively; 6-object of investigation; 7-micro-objective; 10-CCD camera; 11 -personal computer.

\section{Characteristics of the Objects of Investigation}

"Optically thin" ( $\tau d \leqslant 0.1$, "extinction" of radiation by a layer of thickness $d$ ) frozen histological sections of muscular (MT) and skin derma (SD) of human tissue have been investigated. Such samples quite completely represent the main groups of the structured tissues. ${ }^{10}$ The following groups of pathology (muscular dystrophy, SD psoriasis) have been considered. The samples of physiologically normal BT (group "A") and the samples of "conventionally" normal BT, histological sections, taken in the areas which do not correspond to localization of pathological changes in architectonics (group "B") have been used [Figs. 2(a1), (e1), (a2), and (e2)]. From the optical point of view pathological changes of BT structure on the early stages are accompanied by the decrease of the protein fibrils anisotropy substance forming their architectonic nets. ${ }^{17,8}$

The technique of freezing the thin layers of BT to "nitrogen" temperatures provided practically complete identity with their morphological structure both in vivo and in vitro. ${ }^{11}$ From the medical point of view the samples of groups "A" and "B" are practically indistinguishable. Traditional histochemical investigations show no differences in their physiological state. ${ }^{11}$

\section{Experimental Data Analysis and Discussion}

Figure 2 represents coordinate distributions of phase $\delta_{i+1, i}$ [(b1), (f1), (b2), (f2)] and magnitude $\left|V^{2}\left(\Delta r_{i}\right)\right|[(\mathrm{c} 1),(\mathrm{g} 1)$,
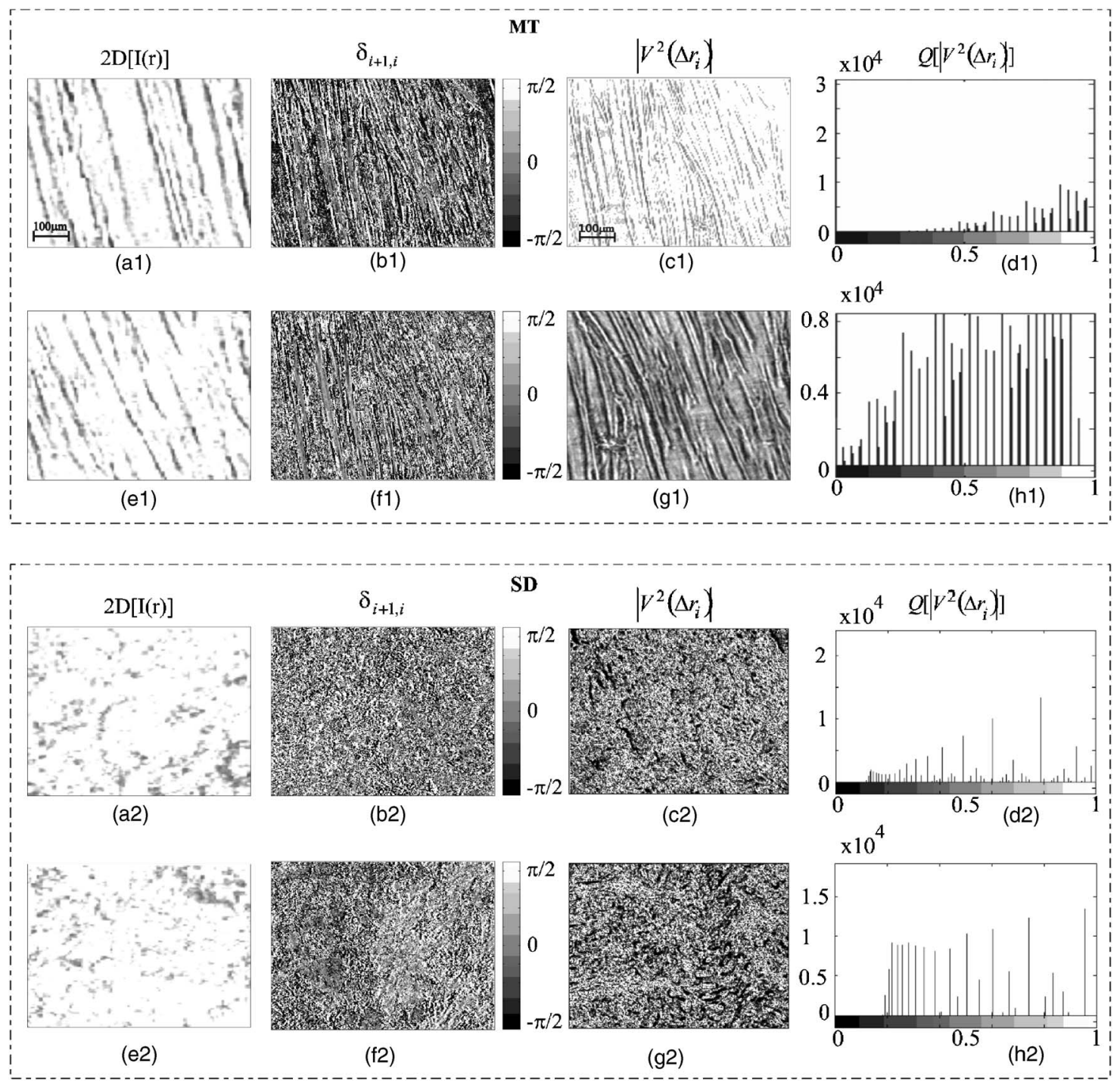

Fig. 2 The CDMP magnitude [(c1), (g1), (c2), (g2)] of MT and SD samples correspondingly. Parts (c1) and (c2) correspond to normal samples, parts (g1) and (g2) to pathologically changed ones. The phase difference between the orthogonal components of polarization is depicted in parts (b1), (f1), (b2), and (f2). Histological images of physiologically normal and pathologically changed samples are presented in parts (a1), (a2) and (e1), (e2) correspondingly. Parts (d1), (h1), (d2), (h2) correspond to statistical distribution of CDMP magnitude. 
Table 1 1st-4th order statistics of CDMP of BT coherent images.

\begin{tabular}{cccccc}
\hline & MT (37 samples) & & & \multicolumn{2}{c}{ SD (34 samples) } \\
\cline { 6 - 6 } \cline { 5 - 6 } Statistic moments & Normal & Pathology & & Normal & Pathology \\
\hline$M_{\vee 2}$ & $0.18 \pm 6 \%$ & $0.96 \pm 9 \%$ & & $0.24 \pm 8 \%$ & $0.35 \pm 10 \%$ \\
$\sigma_{V 2}$ & $0.11 \pm 5 \%$ & $0.67 \pm 10 \%$ & & $0.18 \pm 7 \%$ & $0.41 \pm 9 \%$ \\
$A_{V 2}$ & $67.4 \pm 11 \%$ & $589.7 \pm 16 \%$ & & $89.3 \pm 9 \%$ & $436.217 \% \pm$ \\
$E_{V 2}$ & $324.2 \pm 16 \%$ & $1451.3 \pm 22 \%$ & & $174.9 \pm 13 \%$ & $981.7 \pm 21 \%$ \\
\hline
\end{tabular}

(c2), (g2)] with [(d1), (h1), (d2), (h2)] histograms of physiologically normal and pathologically changed BT coherent images. The analysis of obtained data about coordinate and statistic structure of 2-D distributions of the CDMP parameter of BT speckle images shows that distributions $\left|V^{2}\left(\Delta r_{i}\right)\right|$ of coherent images of all types of physiologically normal BT are rather close in their structure-mainly formed by the areas [Fig. 2(c1), (c2)] with maximally correlated state of polarization $\left|V^{2}\left(\Delta r_{i}\right)\right| \rightarrow 1$. Distributions $\left|V^{2}\left(\Delta r_{i}\right)\right|$ of coherent images of the pathologically changed BT samples are formed by the areas for which the value of CDMP changes within wide limits $\left(0 \leqslant\left|V^{2}\left(\Delta r_{i}\right)\right| \leqslant 1\right)$ [Fig. 2(g1), (g2)]. Corresponding histograms $Q\left[\left|V^{2}\left(\Delta r_{i}\right)\right|\right]$ contain the ensemble of equiprobable extrema for the whole range the CDMP value changes of coherent images.

The results of comparative analysis of statistic moments of the 1st-4th orders of the $\left|V^{2}\left(\Delta r_{i}\right)\right|$ value of coherent images of the groups of physiologically normal $\left(M_{V^{2}}^{*} ; \sigma_{V^{2}}^{*} ; A_{V^{2}}^{*} ; E_{V^{2}}^{*}\right)$ and pathologically changed $\left(M_{V^{2}} ; \sigma_{V^{2}} ; A_{V^{2}} ; E_{V^{2}}\right)$ BT of all types are presented in Table 1.

It can be seen that the values of statistic moments of CDMP images of physiologically normal and pathologically changed BT are sufficiently different. These differences (up to 1 order) are the most pronounced for the 3rd and 4th statistic moments.

\section{Conclusion}

The results of this investigation can be applied in clinical practice in the following ways:

- in vitro: by the use of BT biopsy technique (if it is possible);

- in vivo: by the use of the suggested technique of 2-D analysis of BT images CDMP obtained by means of the techniques of polarization-sensitive optical coherent tomography. ${ }^{12,13}$

\section{References}

1. A. G. Ushenko and V. P. Pishak, "Laser polarimetry of biological tissue. Principles and applications," in Coherent-Domain Optical Methods-Biomedical Diagnostics, Environmental and Material Science, V. Tuchin, Ed., pp. 67-93, Kluwer Academic Publishers, Norwell, MA (2004).

2. F. Gori, "Matrix treatment for partially polarized, partially coherent beams," Opt. Lett. 23, 241-243 (1998).

3. E. Wolf, "Significance and measurability of the phase of a spatially coherent optical field," Opt. Lett. 28, 5-6 (2003).

4. M. Mujat and A. Dogariu, "Polarimetric and spectral changes in random electromagnetic fields," Opt. Lett. 28, 2153-2155 (2003).

5. M. Mujat, A. Dogariu, and G. S. Agarwal, "Interferometric measurement of the degree of polarization and control of the contrast of intensity fluctuations," Opt. Lett. 29, 1539-1541 (2004).

6. J. Ellis and A. Dogariu, "Complex degree of mutual polarization," Opt. Lett. 29, 536-538 (2004).

7. O. V. Angelsky, G. V. Demianovsky, A. G. Ushenko, D. N. Bukcovets, and Yu. A. Ushenko, "Wavelet analysis of two-dimensional birefringence images of architectonics in biotissues for diagnosing pathological changes," J. Biomed. Opt. 9, 679-690 (2004).

8. O. V. Angelsky, A. G. Ushenko, D. N. Burkovets, and Yu. A. Ushenko, "Polarization visualization and selection of biotissue image two-layer scattering medium," J. Biomed. Opt. 10, 679-690 (2004).

9. O. V. Angelsky, N. N. Dominikov, P. P. Maksimyak, and T. Tudor, "Experimental revealing of polarization waves," Appl. Opt. 38, 31123117 (1999).

10. S. C. Cowin, "How is a tissue built?," J. Biomed. Eng. 122, 553-568 (2000).

11. V. G. Naumenko and N. A. Mityaeva, Histological and Topological Research Techniques in Medicine, p. 304, Medicine Publ., Moscow (1980).

12. J. F. de Boer, T. E. Milner, M. J. C. van Gemert, and J. S. Nelson, "Two-dimensional birefringence imaging in biological tissue by polarization-sensitive optical coherence tomography," Opt. Lett. 22, 934-936 (1997).

13. S. Jiao, W. Yu, G. Stoica, and L.-H. Wang, "Optical-fiber-based Mueller optical coherence tomography," Opt. Lett. 28, 1206-1208 (2003) 